\section{Minimal-invasiv statt osteoplastisch-obliterierend}

\section{Wie wirksam und sicher ist bei chronischer Stirnhöhlenentzündung die endoskopisch modifizierte Operation nach Lothrop? In einer Metaanalyse wurden dazu Daten von 612 Patienten ausgewertet.}

L ange Zeit wurden bei chronisch-rezidivierender Sinusitis frontalis als letzte Behandlungsstufe die Stirnhöhlen obliteriert. Dieser Eingriff war mit einer hohen Erfolgsrate, aber auch mit häufigen Komplikationen bis hin zu Duraund Orbitaverletzungen verbunden. Mittlerweile hat die endoskopische Variante der Lothrop-Operation (auch bekannt als Draf III) mit Resektion des Stirnhöhlenbodens, des interfrontalen Septums und des Septums im Bereich der oberen Nasenhaupthöhle zunehmend an Bedeutung gewonnen.

Ein Autorenteam wertete 33 Studien mit 612 Patienten zu dieser Operation im Zeitraum von 1990 bis 2008 aus. Das Durchschnittsalter lag bei 47,9 Jahren, die postoperative Nachbeobachtungszeit betrug im Schnitt 28,5 Monate. In $75,2 \%$ der Fälle erfolgte die Operation wegen chronischer Stirnhöhlenentzündung, bei 21,3\% war eine Mukozele die Indikation für den Eingriff. Als häufigste Komorbiditäten wurden Polyposis nasi (26,8\%), Asthma bronchiale (6,4\%), allergische Rhinitis $(5,1 \%)$ und laryngopharyngealer Reflux (2,6\%) festgestellt. Insgesamt wurde nur in 20,3\% der Fälle nicht bildgestützt operiert, Stents wurden in 6,0\% der Fälle eingesetzt. In über 99\% der Fälle konnten die Patienten innerhalb von 24 Stunden entlassen werden.

Schwere Komplikationen wie (z.B. Pneumocephalus, Liquoraustritt) traten nur bei insgesamt vier Patienten auf. Leichtere Komplikationen wie Hypos- mie, Dehiszenz des Nasenbeins oder Epistaxis waren in 4\% der Fälle zu beobachten. Die Fehlerquote des Eingriffs - definiert als notwendige Revision im Bereich der Stirnhöhlen - lag bei 13,9\%. Rund $82 \%$ der Patienten berichteten von einer Verbesserung ihrer Symptome bis hin zur Beschwerdefreiheit, bei 16,0\% war keine Veränderung feststellbar und nur in 1,2\% der Fälle verschlechterten sich die Symptome.

af

Anderson P et al. Safety and efficacy of the endoscopic modified Lothrop Procedure: a systematic review and meta-analysis.

Laryngoscope 2009; 119: 1828-33

Kommentar: Lothrop beschrieb 1914 das Prinzip der Kontralateraldrainage durch Fortnahme von Anteilen des Septum interfrontale mit der Entfernung des medialen Stirnhöhlenbodens. Kressner (1950), Goodale und Montgomery (1961) berichteten später über die Kontralateraldrainage der Stirnhöhle. Die eigentliche Mediandrainage zur Verbesserung der Drainage nach beidseitiger Stirnhöhlenoperation wurde von Chaput 1905 und Laurens 1907 nach doppelseitiger externer Stirnhöhlenoperation beschrieben.

Wolfgang Draf klassifizierte die intranasalen Zugänge zur Stirnhöhle (Abb.1). Diese Einteilung hat sich in der Praxis sehr bewährt. Die Indikation zu einer Stirnhöhlenoperation nach Draf I-III ergibt sich immer aus der konkreten anatomischen Situation, der Symptomatik und der rhinologischen Anamnese. Wird der Recessus frontalis in typischer Weise durch Infundibular-, Agger nasi- oder suprabullöse Zellen obstruiert, gelingt eine Erweiterung meist durch eine gezielte Abtragung dieser Zellen unter Sicht der 45-Grad-Optik . Bei einem Typ Ilb können Anteile des Stirnhöhlenbodens mit der Stirnhöhlenstanze abgetragen werden. Die Schleimhaut der hinteren Zirkumferenz sollte immer erhalten werden, um zirkuläre narbige Re-Stenosierungen zu vermeiden. Der Rhinologe muss die Mediandrainage in seinem chirurgischen Repertoire haben, sie ist aber eine Methode für Problemfälle, wie etwa entzündliche Rezidive nach Voroperationen, Re-Stenosierungen und Obliterationen des Recessus frontalis. Dennoch kann auch die Median-Drainage in Einzelfällen nicht vor Restenosierungen bewahren. In diesen Fällen besteht nach wie vor die Indikation zur Obliteration der Stirnhöhle beispielsweise über einen osteoplastischen Zugang oder bei Voroperation nach Ritter-Jansen über die alte Narbe. Besonders kleine, schlecht pneumatisierte Stirnhöhlen mit rezidivierenden Abschottungen und Rezidiv-Mukozelen sind hierfür prädestiniert. Wichtig ist, dass die Höhle unter Sicht des Mikroskops und Endoskops zu $100 \%$ entepithelisiert wird. Die Obliteration der Verbindung zur Nase wird belassen und das Cavum frontale mit Bauch-oder Oberschenkelfett aufgefüllt. Eine weitere Methode der Revisionschirurgie ist die KIMTechnik (kontra-ipsi-medial-Drainage) mit Rekonstruktion des Stirnhöhlenbodens mit Conchaknorpel.

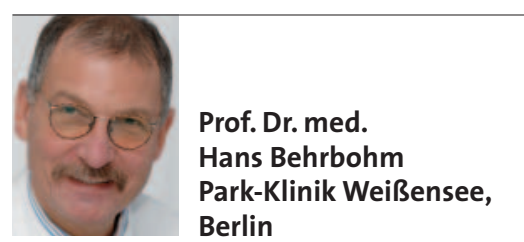

